\title{
TITLE:
}

\section{Folding/unfolding kinetics on a semiflexible polymer chain}

$\operatorname{AUTHOR}(\mathrm{S})$ :

Sakaue, T; Yoshikawa, $\mathrm{K}$

\section{CITATION:}

Sakaue, T ...[et al]. Folding/unfolding kinetics on a semiflexible polymer chain. JOURNAL OF CHEMICAL PHYSICS 2002, 117(13): 6323-6330

\section{ISSUE DATE:}

2002-10-01

URL:

http://hdl.handle.net/2433/49891

\section{RIGHT:}

Copyright 2002 American Institute of Physics. This article may be downloaded for personal use only. Any other use requires prior permission of the author and the American Institute of Physics. 


\title{
Folding/unfolding kinetics on a semiflexible polymer chain
}

\author{
T. Sakaue and K. Yoshikawa \\ Department of Physics, Graduate School of Science, Kyoto University \& CREST, Kyoto 606-8504, Japan
}

(Received 15 April 2002; accepted 11 July 2002)

\begin{abstract}
We studied the kinetic process of a folding/unfolding transition of a long semiflexible polymer chain by Brownian dynamics simulation. A semiflexible chain with an elongated coil conformation is folded into a compact and ordered structure, such as a toroid or rod, through the processes of nucleation and growth. This transition is characterized as a disorder-order transition. The folded morphology is not necessarily the global minimum of free energy, which demonstrates the crucial effect of kinetics on the semiflexible chain folding. The growth process of toroid formation is characterized by a constant growth rate reflecting the dimensionality of a chain, which corresponds well with experimental observations of DNA folding. It is also shown that the path of the unfolding process is much different from that of folding. (C) 2002 American Institute of Physics.
\end{abstract}

[DOI: $10.1063 / 1.1504431]$

\section{INTRODUCTION}

The collapse transition, or coil-globule transition, of a single polymer chain has attracted much interest for several decades. ${ }^{1-6}$ One of the main motivations among the wide range of problems on this subject is to understand the structures and behaviors of biopolymers, such as DNA and proteins, in living cells. The coil-globule transition has usually been considered to be analogous to the gas-liquid transition, where the term globule implies the character of a spherical liquid with minimum surface area. According to the meanfield theory, the order of this phase transition depends on the chain stiffness, i.e., second-order for a flexible chain and first-order-like transition for a stiff chain. ${ }^{1,2}$ On the other hand, recent Monte Carlo studies have shown that a stiff or semiflexible chain exhibits a discontinuous folding transition from the elongated coil into the crystalline or liquid crystalline structure..$^{7-12}$ The folded products show morphologies, such as toroid and rod, similar to those of collapsed DNAs observed by electron microscopy. ${ }^{7,13,14}$

As for the kinetics of the folding transition in a single polymer chain, most of the previous theoretical works treated the process in a flexible chain, where the final product of the transition is a liquidlike sphere (globule). ${ }^{15-26}$ According to the study by de Gennes, the collapse transition is described by a sausage model, where a long flexible polymer is described as a sequences of thermal blobs, and the blobs tend to stick together and take a shape similar to a sausage during the collapse. ${ }^{15}$ Grosberg et al. extended this model to include possible entanglement effects at a late stage in the collapse of a long chain and proposed two-stage kinetics. ${ }^{16}$ This twostage kinetics was indeed observed by Chu et al. using dynamic light scattering in a dilute solution of polystyrene in cyclohexane, ${ }^{27}$ but the interpretation of experimental results is still under dispute at present. ${ }^{28}$ Computer simulation studies have shown that the collapse transition consists of several distinguishable stages, each of which is characterized by a length scale and a time scale. ${ }^{17,18}$ In the very beginning after quenching below the $\theta$ point, numerous local dense clusters are formed. In the subsequent coarsening stage, those clusters grow mainly by unification with smaller ones. In the late stage the process is characterized as a slow approach to the final collapsed state, where the entanglement effect should be significant. A numerical analysis based on the Langevin equation has suggested that the decrease of the gyration radius in the first kinetic stage is ascribed to the change in the large wave-number internal modes. ${ }^{24,25}$ This kinetic feature in the flexible chain is, thus, regarded as a kind of spinodal decomposition.

Thus, nowadays the essential aspects on the collapse kinetics of flexible polymers are well established. Contrary to this, the theoretical scenario on the kinetics of semiflexible chains seems to have not been fully clarified yet. ${ }^{7,11,29-34}$ Such a situation may be unfortunate, because many biological macromolecules, such as DNA, actin and collagen, have a relatively high asymmetry of the ratio $l / \sigma$, where $l$ is the Kuhn length and $\sigma$ the monomer diameter. A flexible chain corresponds to the case of $l / \sigma \simeq 1$. When $l$ is considerably larger than $\sigma$, the long polymer behaves as a semiflexible chain by suitable degree of course graining. A typical example is DNA. In the usual aqueous solution, the Kuhn length of DNA, whose diameter is about $2 \mathrm{~nm}$, is about 100 $\mathrm{nm}$, thus $l / \sigma$ of DNA is about 50 and DNA can be considered as a stiff cylinder in the small length $(\sim \mathrm{nm})$ scale. Most DNAs of biological origin have contour lengths of $\mathrm{mm} \sim \mathrm{cm}$, being several orders larger than their Kuhn length, thus natural DNAs generally behave as semiflexible chains.

As is getting clear that a semiflexible chain undergoes disorder-order transition, it is necessary to study the kinetics of folding transition in relation to the steric structure on the final compact state. Ganazzoli et al. ${ }^{29}$ studied the kinetics of contraction on a freely rotating stiff chain and reported a two-step process, almost without the consideration on the steric structure of the folded chain. Off-lattice Monte Carlo simulation with a circular stiff chain revealed the folding path through metastable states of hairpin conformation. ${ }^{11} \mathrm{Ki}-$ netic aspects of the folding on star/comb heteropolymer ${ }^{34}$ and homopolymer with chemical defects ${ }^{33}$ have also been 
studied with Monte Carlo method. In recent Brownian dynamics simulations using a relatively short stiff linear chain in two ${ }^{32}$ and three dimensions, ${ }^{31}$ it was shown that the stiff chain is folded into a toroid structure through various paths. Since the chain used in these studies had a contour length only several times longer than its Kuhn length, the internal freedom of the chain is highly restricted, consequently the chain should exhibit characteristics between those of a semiflexible and a stiff chain. To get an insight into the folding kinetics of an actual semiflexible chain, i.e., how a long and spatially fluctuating coil is folded into an ordered structure without forming defects and entanglements in the thermal fluctuation, a study on a longer chain is essential. In the present study, we have performed the Brownian dynamics simulation to get deeper insight on the folding scenario on a linear semiflexible macromolecule.

\section{MODEL}

We consider a single linear polymer chain made of $N$ effective monomeric units of diameter $\sigma$. All adjacent monomeric units are connected by the harmonic bonding potential,

$$
U_{\text {bond }}=\frac{k T}{2 \sigma^{2}} \sum\left(\left|\mathbf{r}_{i}-\mathbf{r}_{i+1}\right|-\sigma\right)^{2},
$$

where we choose a relatively large spring constant, $k=400$, to keep the bond length at a nearly constant value $\sigma$, and $T$ is the temperature. We set the Boltzmann constant $k_{B}$ to unity throughout this paper.

The chain stiffness is modeled through the bending potential depending on the angle between adjacent bond vectors,

$$
U_{\text {bend }}=\kappa T \sum\left(1-\frac{\left(\mathbf{r}_{i}-\mathbf{r}_{i-1}\right)\left(\mathbf{r}_{i+1}-\mathbf{r}_{i}\right)}{\sigma^{2}}\right)^{2},
$$

where $\kappa$ is the bending parameter. Considering that the bond length is nearly constant, $U_{\text {bend }}$ is approximately equal to $\kappa T \Sigma(1-\cos \theta)^{2}$.

To model the solvent quality the short-range interaction between nonadjacent monomeric units is represented by a Morse potential,

$U_{\text {int }}=\epsilon T \sum\left[\exp \left\{-2 \alpha\left(\frac{r_{i}-r_{0}}{\sigma}\right)\right\}-2 \exp \left\{-\alpha\left(\frac{r_{i}-r_{0}}{\sigma}\right)\right\}\right]$,

where we set $\alpha=6, r_{0} / \sigma=(\log 2 / \alpha)+1$, and $\epsilon$ is a parameter for the interaction strength, which we set as $\epsilon=0.75$ for the folding simulation. We have confirmed that other choices for the effective potential (e.g., Lennard-Jones, square well, etc.) do not affect the following results, when an appropriate ratio of hard core repulsive and attractive ranges is adopted.

We adopted the Langevin equation which is suitable for the study of dynamics of a coarse grained model. By neglecting the hydrodynamics interaction for simplicity, the equation for the motion of a model polymer chain in thermal and viscous medium is described by

$$
m \frac{d^{2} \mathbf{r}_{i}}{d t^{2}}=-\gamma \frac{d \mathbf{r}_{i}}{d t}+\mathbf{R}_{i}(t)-\frac{\partial U}{\partial \mathbf{r}_{i}},
$$

where $m, \gamma$ are mass and friction constant of monomeric units, respectively. $U$ is the internal potential, $U=U_{\text {bond }}$ $+U_{\text {bend }}+U_{\text {int }}$, and $\boldsymbol{R}_{i}$ is a Gaussian white noise on the $i$ th monomeric unit:

$$
\left\langle\boldsymbol{R}_{i}(t)\right\rangle=0, \quad\left\langle\boldsymbol{R}_{i}(t) \boldsymbol{R}_{j}\left(t^{\prime}\right)\right\rangle=6 \gamma T \delta_{i, j} \delta\left(t-t^{\prime}\right) .
$$

We studied a polymer chain of contour length $L$ $=512 \sigma$. The bending parameter was chosen as $\kappa=60$, indicating that the persistence length $\lambda / \sigma=9.6 \pm 0.15$. These parameters have been adopted so as to satisfy the relationship; $\sigma \ll l \approx 2 \lambda \ll L$. For the statistical data we performed independent 40 calculations from different initial conditions. Hereafter we measure all lengths, energies and time in units of $\sigma$, temperature $T$ and $\tau=\gamma \sigma^{2} / T$, respectively.

\section{RESULTS}

\section{A. Folded products}

Figure 1 shows typical snapshots of the folded products with the two different morphologies, toroid and rod, together with the histograms of the mean value of internal potential normalized by the number of monomeric units $U / N$, radius of gyration $R_{g}$, and the maximum distance between monomeric units $R_{\text {max }}=\max _{\{i, j\}}\left\{\left|\mathbf{r}_{i}-\mathbf{r}_{j}\right|\right\}$. Both toroid and rod are stable, and are separated by a enough free energy barrier under the present conditions.

Figure 1 shows that the toroid structure has lower internal potential than the rod structure, suggesting that the toroid structure may correspond to the global minimum of free energy. The wide distribution of the rod in all of the histograms indicates the presence of multiple local free energy minima. On the other hand, the distribution is rather narrow for the toroid. The maximum distance between monomeric units, $R_{\max }$, corresponds to the outer diameter of the toroid and from Fig. 1 we see that it is almost the same as the Kuhn length of the polymer coil, which is consistent with the observed toroid size of DNA. ${ }^{35,36}$

\section{B. Folding kinetics}

As typical examples for the toroid and rod formation, Figs. 2(a) (toroid) and 3(a) (rod) shows the time development of the normalized internal potential $U / N$ and three other quantities, $P, S_{1}$, and $S_{2}$. The parameter $P$ is the ratio of monomeric units which have at least one neighbor and is defined as

$$
P=\Sigma_{i} \frac{\rho(i)}{N}=\Sigma_{i} \frac{1-\prod_{|i-j|>\lambda}\left(1-\rho_{i, j}\right)}{N},
$$

where $\rho_{i, j}$ is an indicator of the pair contact:

$$
\rho_{i, j}= \begin{cases}1 & \left(\text { if }\left|\boldsymbol{r}_{i}-\boldsymbol{r}_{j}\right|<r_{c}\right), \\ 0 & \text { (otherwise) }\end{cases}
$$




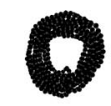

toroid
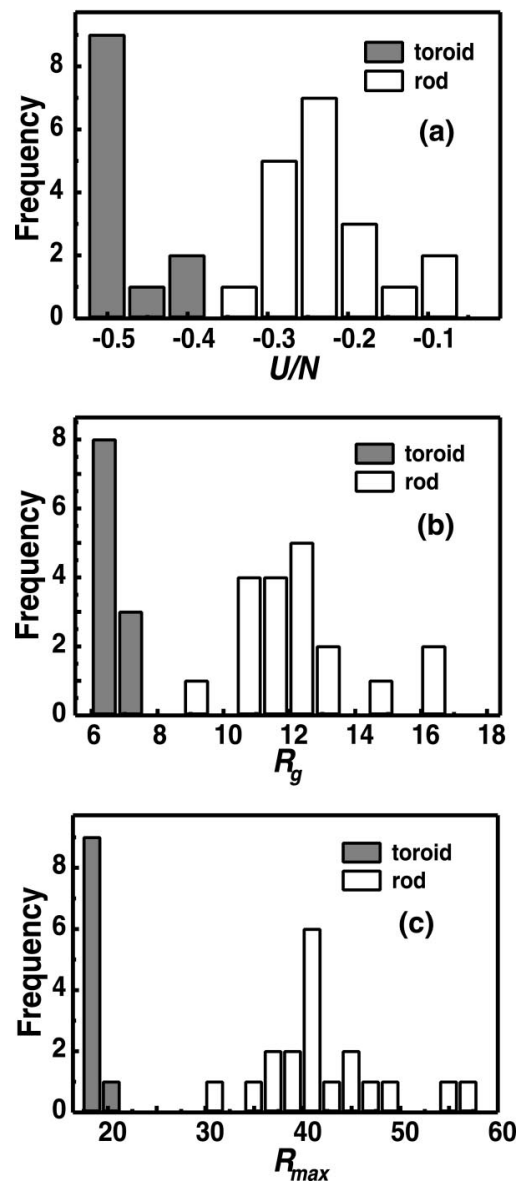

FIG. 1. A snapshot of toroid and rod structure and histograms of (a) normalized internal potential $U / N$, (b) gyration radius $R_{g}$, (c) maximum distance between monomeric units $R_{\max }=\max _{\{i, j\}}\left\{\mathbf{r}_{i}-\mathbf{r}_{j}\right\}$ in the folded state. To obtain the mean value from each trial, the quantity was sampled after the completion of the folding transition, where it was confirmed that measured quantities did not show any substantial change except for the fluctuation around the mean values. The standard deviation is on the order of the bin width.

In the following we set $r_{c}=2$. The parameters $S_{1}$ and $S_{2}$ indicate the degree of local bond orientational ordering, and are calculated from the average operation on $\eta(i)$, which is defined as

$$
\begin{aligned}
& \eta(i)=\left\{\begin{array}{l}
\frac{\sum_{|i-j|>\lambda} \eta_{i, j}}{\sum_{|i-j|>\lambda} \rho_{i, j}} \quad(\rho(i)=1), \\
0 \quad \text { (otherwise) },
\end{array}\right. \\
& \eta_{i, j}=\frac{1}{2}\left(3 \cos ^{2} \theta_{i j}-1\right) \rho_{i, j} .
\end{aligned}
$$

The notation $\eta_{i, j}$ is an indicator of orientational ordering between two contacting bonds and $\theta_{i j}$ is the angle between them. To analyze the local structure around monomeric units due to the nonbonded long range interactions, the monomeric units within persistent length $\lambda$ are not included to calculate (a)
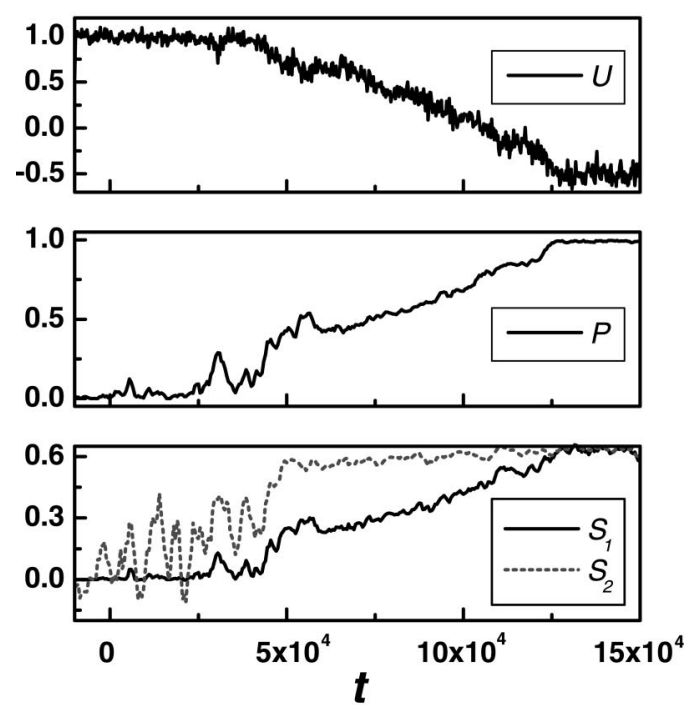

(b)

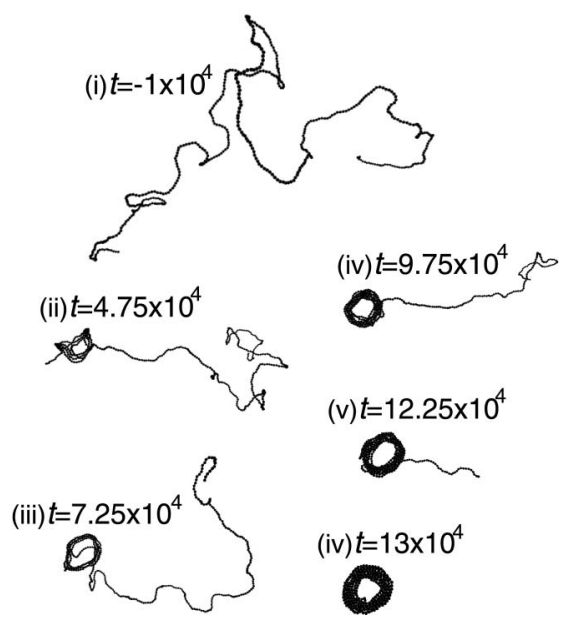

FIG. 2. (a) The time development of (top) normalized internal potential $U / N$, (middle) the ratio of monomeric units which have at least one neighbor $P$, (bottom) the degree of the local bond orientational ordering $S_{1}$ and $S_{2}$ for the toroid formation. (b) A sequence of conformational change during the folding process for the toroid formation.

$\rho(i), \quad \eta(i)$. The order parameter $S_{1}$ is the average of $\eta(i)$ over all monomeric units, thus, indicates the ratio of the orientational ordering of the polymer chain

$$
S_{1}=\langle\eta(i)\rangle=\frac{\sum_{i} \eta(i)}{N} .
$$

The other order parameter $S_{2}$ is the average of $\eta(i)$ only on the monomeric units having at least one neighbor

$$
S_{2}=\langle\eta(i)\rangle_{(\rho(i) \neq 0)} .
$$

Therefore $S_{2}$ reflects the presence or absence of the orientational ordering along a chain. Figure 2(b) shows the conformations of polymer chain corresponding to Fig. 2(a). After changing the solvent quality from good to poor, the chain remains in the coil state for a while, suggesting that the coil conformation is a metastable state. Owing to the effective attractive interaction between monomeric units, pairs of monomeric units stick to each other for a short time during 


\section{(a)}
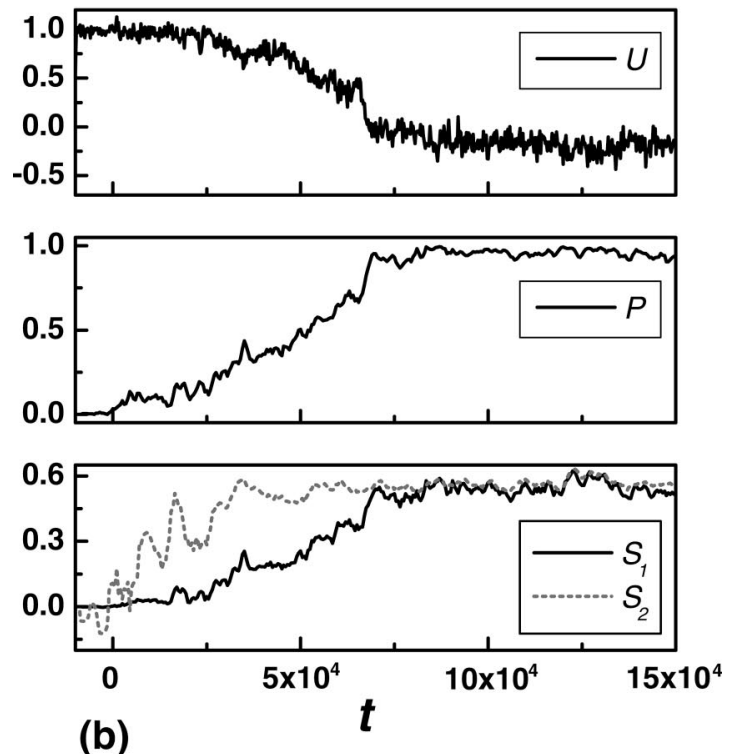

(b)

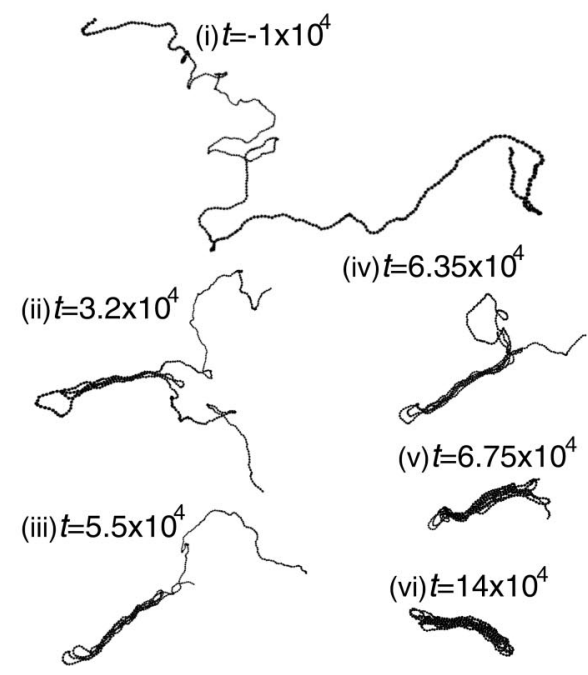

FIG. 3. (a) The time development of (top) normalized internal potential $U / N$, (middle) the ratio of monomeric units which have at least one neighbor $P$, (bottom) the degree of the local bond orientational ordering $S_{1}$ and $S_{2}$ for the rod formation. (b) A sequence of conformational change during the folding process for the rod formation.

thermal agitation, however, such pairs soon separate. As for the manner of monomers contacts, $S_{2}$ indicates that the direction of contacting bonds are not isotropic but prefer oriented alignment, though the fluctuation is large due to the small number of monomeric pairs satisfying $\left|\mathbf{r}_{i}-\mathbf{r}_{j}\right|<r_{c}$ in this stage. If a large enough doughnut shaped nucleus (critical nucleus) is formed at a certain occasion, the remaining coil part is pulled into the nucleus in order and finally the toroid structure is formed. It should be noted that such nucleation accompanies the increase of $S_{2}$, indicating high orientational ordering in the nucleus. The typical characteristic of toroid formation is the almost constant speed of the growth process as is shown in Fig. 2.

Figure 3(b) shows the polymer chain conformations corresponding to Fig. 3(a). Similar to the toroid formation, the coil state survives for some time after quenching and, then, a rod shaped critical nucleus with an orientational order is formed in a stochastic way. The typical growth process of rod formation consists of almost flat parts or relatively gentle slopes and abruptly changing parts [Fig. 3(a)]. The structures like Fig. 3(b)(ii), (iii), where the remaining coil part reaches the edge of the rod during the growth process, have a finite lifetime, since the free energy barrier arising from the difficulty in bending the stiff chain should be overcome to grow further. We observed that the growth from this metastable state consists of two processes. The first process is the abrupt adsorption of a portion of the coil part into the rod shaped nucleus in a random and disordered way. After that, the second slow process follows where the monomeric units inside the rod shaped nucleus are rearranged to lower the free energy.

It is noted that the above descriptions are the typical examples which are often observed, but of course the actual folding process is more diverse. The following processes were observed: (i) the rod shaped critical nucleus is formed, however, before the remaining coil part is pulled into the nucleus, a hole appears in the center of the nucleus to change into a doughnut shape which grows to be the toroid structure (2/40): (ii) the large rod shaped nucleus with multiple loop parts are formed, which turns into the toroid by bending $(2 / 40)$. In both cases the generated toroids have many hairpin defects.

\section{Unfolding process}

We now discuss the reverse process, i.e., unfolding from the condensed, ordered phase to the dispersed coil phase. In contrast to the folding process where the quasi-onedimensionality of the chain is essential, the melting can only begin at the surface on a three-dimensional condensed object. Thus we can easily expect that the kinetic process of the unfolding transition is much different from that of the folding ${ }^{30}$ as is usual in a first-order phase transition.

To see the character of the unfolding process, Fig. 4(a) shows an example of the time development of the same quantities monitored for the folding process at $\epsilon=0.6, \kappa$ $=60$, where the starting conformation is a toroid obtained in the folding simulation $(\epsilon=0.75, \kappa=60)$. Since the condensed phase is metastable in this condition, the monomeric units on the surface try to dissolve, or to evaporate. Due to the connectivity of the polymer chain, however, the small fraction of melted monomeric units soon go back into the metastable toroid, and it cannot be unfolded to a coil state until a large enough fraction of monomeric units evaporate. Once the metastable state becomes unstable, the whole chain is unfolded in an abrupt way. This point is in sharp contrast to the folding process, where the new equilibrium phase appears at some part of the chain and the ordered structure is formed by the propagation of the boundary between this new equilibrium phase and the metastable phase. This difference is clearly seen by comparing the time development of the order parameters $S_{1}$ and $S_{2}$ in folding and unfolding processes [Figs. 2(a) and 4(a)]. Note that the difference in the pathway between folding and unfolding processes has been 
(a)
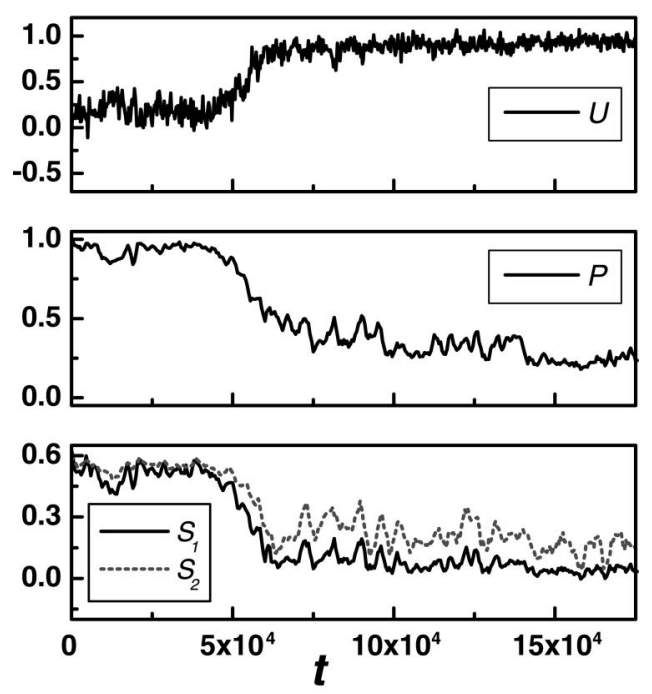

(b)

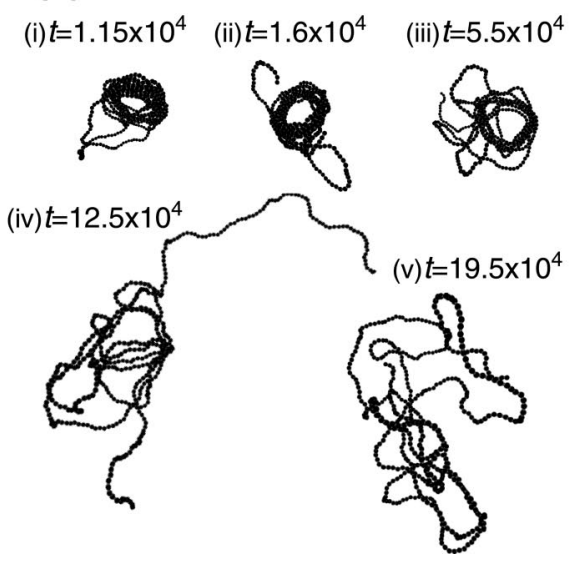

FIG. 4. (a) The time development of (top) normalized internal potential $U / N$, (middle) the ratio of monomeric units which have at least one neighbor $P$, (bottom) the degree of the local bond orientational ordering $S_{1}$ and $S_{2}$ for the unfolding process at $\epsilon=0.6, \quad \kappa=60$. As an initial condition we used a toroid conformation obtained in the folding simulation $(\epsilon=0.75, \kappa$ $=60$ ). (b) A sequence of conformational change during the unfolding process.

actually observed through the experiment on the direct observation of a single DNA molecule. ${ }^{37}$

It should be noted that entanglements are sometimes formed in the early stage of the unfolding. Figure 4 is an example of such processes. Once entanglements are formed, they are usually preserved during the successive stage of the unfolding because of the nonphantom nature of the real chain. Since it is very difficult to untie the entanglement in the swelled coiled state, this topologically constrained coil [Fig. 4(b)(iv),(v)] has a very long lifetime.

\section{DISCUSSION}

\section{A. Nucleation}

As described above, a coil state continues as a metastable state with a finite lifetime after changing the solvent quality from good to poor. This indicates that the free energy
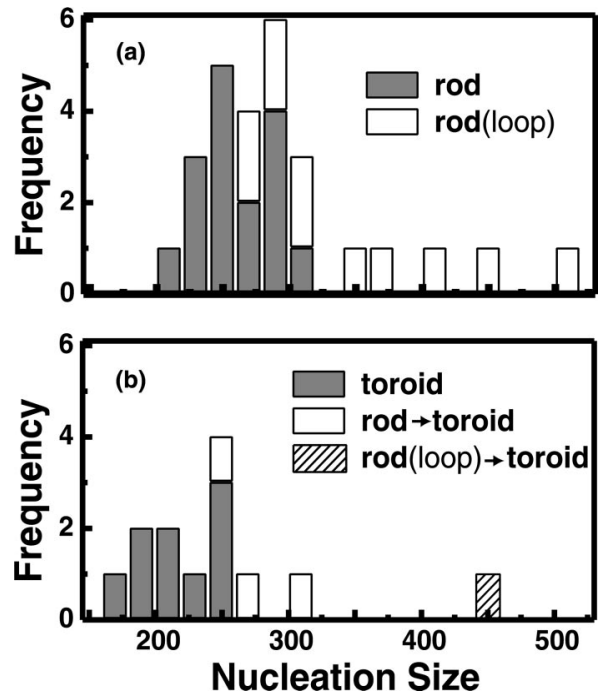

FIG. 5. Histograms of the size of the critical nucleus in which (a) the rod, (b) the toroid are formed as the final product. The word size means the number of monomeric units needed for the formation of critical nucleus, where the monomeric units in loop sections, if any, are also included, and distinguished by the column type. The arrows in (b) signify that the generated rod shaped nucleus changed into a toroid during successive folding.

as a function of order parameter has a bimodal profile near the transition point under present conditions, i.e., the transition is first-order.

As described in Sec. III, kinetics is a crucial factor for the folded morphologies, which is almost determined at the nucleation stage. To argue the relative probabilities for the appearance of the toroid and rod shaped critical nuclei, it is, in principle, possible to calculate the nucleation free energy barrier for each case. However this is insufficient in the case of the polymer chain whose kinetic pathway in the phase space is strongly restricted due to the fact that all monomeric units are connected into a chain and any chain section cannot cross another. Figure 5 shows a histogram of the critical nucleus size (the number of monomeric units required to form a critical nucleus) for the toroid and rod formation. The critical nucleus was expediently judged to be formed when the interaction energy $U_{\text {int }} / N$ falls below -0.25 irreversibly. This corresponds to the point where the parameter $S_{2}$ becomes a high stable value [Figs. 2(a) and 3(a)]. As for the definition of the size, when the critical nucleus includes some loop structures, the loop parts are also included in the number of monomeric units required to form the critical nucleus. There is a tendency that the critical nucleus size of a toroid is smaller than that of a rod. In addition, when the critical nucleus includes some loop parts, it is always rod shaped, and most of them are folded into the rod. This result indeed shows that the diversity of the pathway toward the rod shaped nucleus is a crucial factor in determining the type of nucleation, which eventually affects the final folded morphology. It is noted that the relative probabilities for the toroid and rod appearance may depend on the degree of supercooling. Further careful study is necessary to make clear the relationship of the quench depth on the final folded morphology.

According to the experiments in DNA collapse toroids 


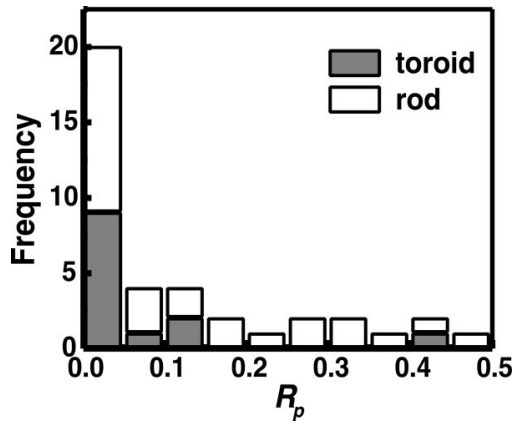

FIG. 6. Histogram of $R_{p}$, the position where the critical nucleus was formed along the chain. The two cases, corresponding to the rod and the toroid formation, are distinguished by different column type.

are the most common morphology in the usual experimental conditions. Whereas some condensing agents have been reported to tend to produce more rod morphology. ${ }^{13,14}$ It has been shown that the folded equilibrium morphologies are determined by the competing effects of chain stiffness and attractive interaction, and also by chain length. ${ }^{9,10,12}$ The present result demonstrates the crucial effect of the kinetics on the folded morphologies for a semiflexible chain. ${ }^{711}$ It should be noted that the polyelectrolyte nature of DNA may also affect the relative stabilities of toroid and rod shaped nuclei, and thus the corresponding free energy barrier.

Figure 6 shows the position where the critical nucleus is formed along the chain. The position $R_{p}$ is defined by

$$
R_{p}=\left\{\begin{array}{l}
\frac{L_{b}}{L_{a}+L_{b}} \quad\left(\text { if } \frac{L_{b}}{L_{a}+L_{b}} \leqslant 0.5\right), \\
1.0-\frac{L_{b}}{L_{a}+L_{b}} \quad \text { (otherwise), }
\end{array}\right.
$$

where $L_{a}$ is the length of free tail (in a coil state) on one side of the nucleus and $L_{b}$ is the same quantity on the other side. We see that there is a high probability that the nucleus is formed at the chain end, reflecting the large freedom in thermal motion. The same tendency was reported by the direct observation of the nucleation and growth of giant DNA molecules using fluorescence microscopy. ${ }^{38}$ In this experiment the probability of nucleation dependence on the base-pair sequence was also reported, which indicates the importance of the chain stiffness on the folding kinetics. The special role of the chain end in the folding kinetics of the flexible chain has also been reported. ${ }^{19,20}$ Thus the folding kinetics is expected to be modified in circular chain for both flexible and semiflexible polymer.

\section{B. Growth process}

It is well known that the growth rate depends on the size (curvature) of the nucleus in the usual diffusion controlled crystal growth in three-dimensional space such as liquid droplet formation from supercooled gas. ${ }^{39}$ In contrast, as described in Sec. III B, the typical kinetics of toroid formation is characterized by nearly constant growth speed after nucleation. In fact, such a feature in the folding transition is seen in a single DNA molecule observation using fluorescence microscopy. ${ }^{37,38,40}$ Figure 7 exemplifies the time course of (a)
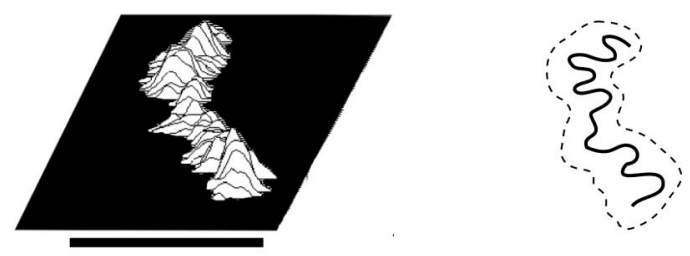

(b)
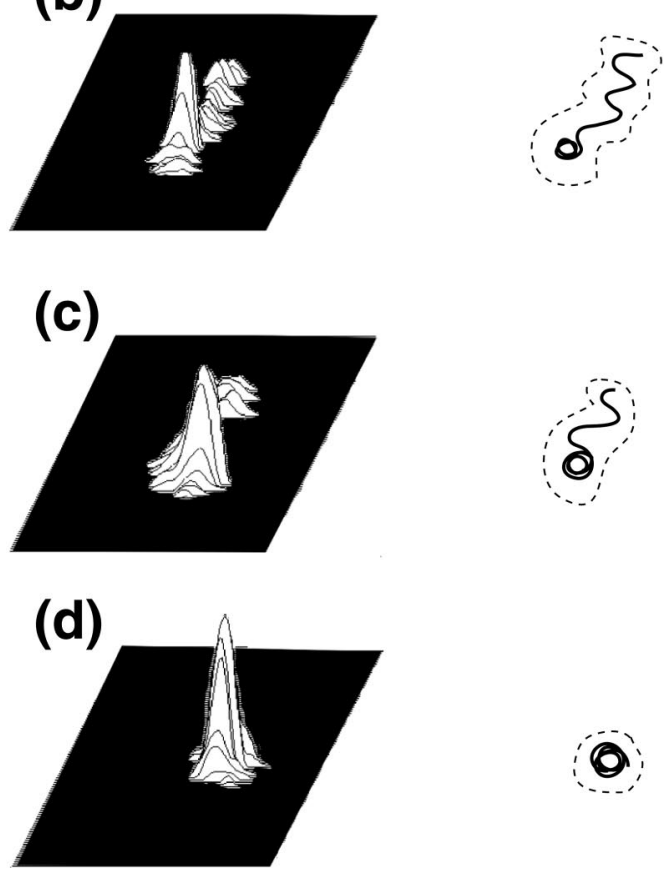

FIG. 7. Experimental observation on nucleation and growth in a single T4 DNA molecule (Ref. 38). Quasi-three-dimensional plot of the fluorescent intensity distribution and the schematic representation of the DNA conformation during the folding process. (a) The metastable coil has a lifetime on the order of several tens of minutes. (b) During thermal agitation a bright spot spontaneously appears at a certain point on the chain. (b)-(d) The fluorescence intensity on the spot gradually increases. The time interval of these figures is $2 \mathrm{~s}$ except for $6 \mathrm{~s}$ between (a) and (b). The scale bar represents $10 \mu \mathrm{m}$.

the fluorescence intensity during the folding transition of a single T4 DNA molecule induced by the addition of polyethylene glycol. After the spontaneous formation of the critical nucleus, it grows at a constant speed. Then, what is a suitable order parameter to characterize this dynamic process? We are now treating a one-dimensional string embedded in threedimensional real space. The result of our simulation (Fig. 2) together with the experimental observation as in Fig. 7 indicates the appearance of one-dimensional nature on the process of growth of the folded compact part along a chain. Thus, by considering a quasi-one-dimensional nature of a polymer chain, we adopt the local segment density $\phi(x, t)$ as a function of the position along the chain $x$ and of time $t$, where $\phi(x, t)$ should be smoothed out to be a quasimacroscopic parameter from the average operation on $\rho(i)$ over many or at least several monomeric units. In the collapsed part, $\phi(x, t)$ is large, while its value is low in the coil part. Figure 8 shows an example of the time development of $\phi(x, t)$ calculated from the simulation (the same run as in 


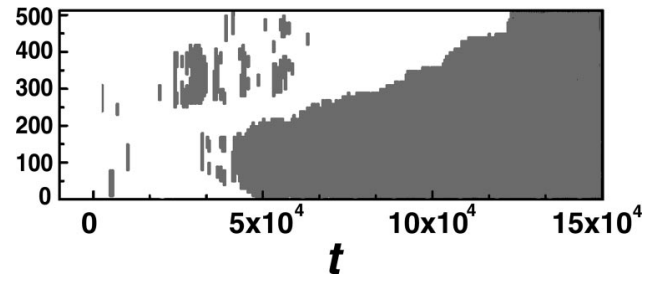

FIG. 8. The time development of the two valued local segment density profile $\phi(x, t)$ as a function of the position along the chain $x$ and of time $t$, where the white and shaded regions represent the coil part and dense condensed part, respectively. $\phi(x)$ is calculated from $\rho(i)$, which reflects the presence $[\rho(i)=1]$ or absence $[\rho(i)=0]$ of other monomeric units in the close vicinity of $i$ th monomeric unit [Eq. (6)]. The rather rough spatial profile $\rho(i)$ is smoothed to produce the smooth profile $\phi(x)$. We have performed the smoothing using neighboring 10 monomeric units.

Fig. 2). Since the density profile during the growth process is found to be approximated by a steplike function, we set the threshold, above (below) which the segments around $x$ were judged to belong to the condensed (coil) phase. In the Fig. 8 the shaded and white parts represent the dense condensed part and coil part, respectively. This figure indeed exemplifies the usefulness of $\phi(x, t)$ in the description of the dynamics of the spatial inhomogeneity along the chain. It should be noted that the density $\phi(x, t)$ is not a conserved quantity in the present description. Instead of $\phi(x, t)$, we can also adopt the degree of local orientational ordering [smoothed-out $\eta(i)]$ as an order parameter, since they are coupled as has been shown in the Sec. III B.

The time development of $\phi(x, t)$ is given by the following:

$$
\frac{\partial \phi(x)}{\partial t}=-L \frac{\delta F\{\phi(x)\}}{\delta \phi(x)},
$$

where $F\{\phi\}$ is Ginzburg-Landau free energy;

$$
F\{\phi(x)\}=\int d x\left(f\{\phi(x)\}+\frac{C}{2}\left(\frac{\partial \phi}{\partial x}\right)^{2}\right),
$$

where we do not consider the presence of the long range interactions along the one-dimensional chain explicitly, which indeed are indispensable for the folding but not for the growth process. The first term in the integral is the free energy density on $x$, and the second term describes the effect of a spatial gradient on the order parameter. Thus we obtain the following equation:

$$
\frac{\partial \phi(x)}{\partial t}=-L\left(\frac{\partial f(\phi)}{\partial \phi}-C \frac{\partial^{2} \phi}{\partial x^{2}}\right) .
$$

This differential equation has the solution of a traveling wave with a constant speed. For simplicity let us consider the fourth-order polynomial as $f(\phi)$ to describe the bistable system, $\partial f(\phi) / \partial \phi$ is represented by

$$
\frac{\partial f(\phi)}{\partial \phi}=a\left(\phi-\phi_{1}\right)\left(\phi-\phi_{2}\right)\left(\phi-\phi_{0}\right),
$$

where $\phi_{1}$ and $\phi_{2}$ correspond to the order parameter of the (meta)stable state, and $\phi_{0}$ is that of the unstable state. Under the boundary condition $\phi(-\infty, t)=\phi_{1}, \phi(+\infty, t)=\phi_{2}$, the traveling speed of the boundary, in other words the growth rate, is calculated

$$
v=L \sqrt{\frac{a C}{2}}\left(2 \phi_{0}-\phi_{1}-\phi_{2}\right) .
$$

Though the length of real polymer chain is finite, this discussion may be useful after a large enough size of nucleus is formed. Equation (17) is represented by using the difference of the free energy density in the coil and condensed states.

$$
v=L \sqrt{\frac{C}{2 a}} \frac{12}{\left(\phi_{1}-\phi_{2}\right)^{3}}\left(f\left(\phi_{1}\right)-f\left(\phi_{2}\right)\right) .
$$

Thus, the growth rate is constant and increases with the degree of supercooling. As the number of statistical segments in the individual polymer chain is not so large, the thermal fluctuation affects it strongly, consequently the observed growth speed has some distribution in the actual growth process.

The above discussion relies on the following two hypotheses: (1) a polymer chain is a quasi-one-dimensional system, thus, the boundary between the condensed nucleus and the coil part is a "point" though the polymer chain is embedded in three-dimensional space. (2) The condensed phase is spatially homogeneous. For the growth process of toroid formation, both conditions are well satisfied within the coarseness of the present description. However, as discussed in Sec. III B, instead of constant growth we observed stepwise growth process for rod formation, since the second condition is violated. It should also be noted that it is sometimes observed during the growth process that the free segments in the coil part which are not adjacent to the nucleus make contact with the nucleus by accident due to the presence of the long range interactions. Such events make the observed kinetics deviate from the above simple argument with a single order parameter.

\section{CONCLUSION}

We have shown that a single semiflexible polymer chain is folded through nucleation and growth into distinguishable ordered morphologies; one is a stable toroid and the other is a (meta)stable rod under the present conditions. Which type of morphologies is produced is mostly decided at the nucleation stage. Previous studies by theory ${ }^{30}$ and simulation ${ }^{11,31,32}$ have revealed the evidence for the metastability and competition between different conformation in the folding of a short stiff chain. Here, we would like to emphasize the novel characteristics in a "long semiflexible" chain different from a "short stiff" chain (more precisely, these are distinguished by the ratio $L / l$ ). From our result, it becomes evident that to form a critical nucleus during thermal fluctuation, about 200 monomeric units (more than 10 Kuhn segments) are required at least (Fig. 5). Thus it is almost impossible to form a natural critical nucleus for a short stiff chain. In the case of a short stiff chain, the possible pathways are the uniform chain shrinking or to form extend conformation (rod or racket) with a few stems at first, which is dynamical intermediate with a finite lifetime. Then such structures are 
likely to be folded into the stable toroid, because the number of monomer contact is small, whereas the bending energy penalty is rather large.

In the case of toroid formation, the growth process is characterized by its constant growth rate reflecting the quasione-dimensionality of the polymer chain. We expect that if the quenching is too deep to lose the stability of a coil state, the folding process should be different and show some similar aspects to that of a flexible chain, especially in the early stage. Indeed, if we set $\epsilon>0.9$ at $t=0$, internal potential $U$ starts to decrease immediately without the induction time, indicating the regime of spinodal decomposition. The kinetic pathway of unfolding is markedly different from that of folding and does not accompany the transient spatial inhomogeneity along the chain. It is noted that such a difference corresponds well to the experimental observation on the folding/ unfolding transition in a single DNA molecule. ${ }^{37}$ It should be noted that the difference in the kinetic pathways for folding and unfolding transitions manifests its significance when we consider the mechanism of molecular machinery, since motor proteins are expected to exhibit a type of order-disorder transition between different condensed phases during the working cycle.

It should be emphasized that such features of folding kinetics at the single molecule level cannot be observed using the conventional experimental techniques of polymer science, such as light scattering, spectroscopies, and sedimentation, where the signal is smoothed out in the ensemble.

We expect that the kinetic effect during the folding/ unfolding observed in the semiflexible homopolymer may manifest its significance for more complicated polymers such as branched polymers, copolymers and also proteins.

\section{ACKNOWLEDGMENT}

The authors thank Dr. Y. Matsuzawa for providing the experimental data (Fig. 7).

${ }^{1}$ I. M. Lifshitz, A. Yu. Grosberg, and A. R. Khokhlov, Rev. Mod. Phys. 50, 683 (1978).

${ }^{2}$ A. Yu. Grosberg and A. R. Khokhlov, Statistical Physics of Macromolecules (American Institute of Physics, New York, 1994).

${ }^{3}$ P. G. de Gennes, Scaling Concepts in Polymer Physics (Cornell University Press, Ithaca, 1979).

${ }^{4}$ C. B. Post and B. H. Zimm, Biopolymers 21, 2413 (1982).

${ }^{5}$ G. Swislow, S. Sun, I. Nishio, and T. Tanaka, Phys. Rev. Lett. 44, 796 (1980).
${ }^{6}$ M. Nakata and T. Nakagawa, Phys. Rev. E 56, 3338 (1997).

${ }^{7}$ H. Noguchi, S. Saito, S. Kidoaki, and K. Yoshikawa, Chem. Phys. Lett. 261, 527 (1996).

${ }^{8}$ J. P. K. Doye, R. P. Sear, and D. Frenkel, J. Chem. Phys. 108, 2134 (1998).

${ }^{9}$ H. Noguchi and K. Yoshikawa, J. Chem. Phys. 109, 5070 (1998).

${ }^{10}$ V. A. Ivanov, W. Paul, and K. Binder, J. Chem. Phys. 109, 5659 (1998).

${ }^{11}$ Yu. A. Kuznetsov and E. G. Timoshenko, J. Chem. Phys. 111, 3744 (1999).

${ }^{12}$ V. A. Ivanov, M. R. Stukan, V. V. Vasilevskaya, W. Paul, and K. Binder, Macromol. Theory Simul. 9, 488 (2000).

${ }^{13}$ G. E. Plum, P. G. Arscott, and V. A. Bloomfield, Biopolymers 30, 631 (1990).

${ }^{14}$ V. A. Bloomfield, Curr. Opin. Struct. Biol. 6, 334 (1996).

${ }^{15}$ P. G. de Gennes, J. Phys. (Paris) 46, L639 (1985).

${ }^{16}$ A. Yu. Grosberg, S. K. Nechaev, and E. I. Shakhnovich, J. Phys. (Paris) 49, 2095 (1988).

${ }^{17}$ A. Byrne, P. Kierman, D. Green, and K. A. Dawson, J. Chem. Phys. 102, 573 (1995).

${ }^{18}$ Yu. A. Kuznetsov, E. G. Timoshenko, and K. A. Dawson, J. Chem. Phys. 103, 4807 (1995).

${ }^{19}$ B. Ostrovsky and Y. Bar-Yam, Europhys. Lett. 25, 409 (1994).

${ }^{20}$ B. Ostrovsky and Y. Bar-Yam, Biophys. J. 68, 1694 (1995).

${ }^{21}$ A. Buguin, F. Brochard-Wyart, and P. G. de Gennes, C. R. Acad. Sci., Ser. IIb: Mec., Phys., Chim., Astron. 322, 741 (1996).

${ }^{22}$ L. L. Klushin, J. Chem. Phys. 108, 7917 (1998).

${ }^{23}$ A. Halperin and P. M. Goldbart, Phys. Rev. E 61, 565 (2000).

${ }^{24}$ E. G. Timoshenko, Yu. A. Kuznetsov, and K. A. Dawson, J. Chem. Phys. 102, 1816 (1995)

${ }^{25}$ Yu. A. Kuznetsov, E. G. Timoshenko, and K. A. Dawson, J. Chem. Phys. 104, 3338 (1996).

${ }^{26}$ E. Pitard and H. Orland, Europhys. Lett. 41, 467 (1998).

${ }^{27}$ B. Chu, Q. Ying, and A. Yu. Grosberg, Macromolecules 28, 180 (1995).

${ }^{28}$ P. W. Zhu and D. H. Napper, J. Chem. Phys. 106, 6492 (1997); M. Nakata and T. Nakagawa, ibid. 110, 2703 (1999).

${ }^{29}$ F. Ganazzoli, R. La Ferla, and G. Allegra, Macromolecules 28, 5285 (1995).

${ }^{30}$ Yu. A. Kuznetsov, E. G. Timoshenko, and K. A. Dawson, J. Chem. Phys. 105, 7116 (1996).

${ }^{31}$ H. Noguchi and K. Yoshikawa, J. Chem. Phys. 113, 854 (2000),

${ }^{32}$ B. Schnurr, F. C. Mackintosh, and D. R. M. Williams, Europhys. Lett. 51, 279 (2000).

${ }^{33}$ D. Hu, J. Yu, K. Wong, B. Bagchi, P. J. Rossky, and P. F. Barbara, Nature (London) 405, 1030 (2000).

${ }^{34}$ E. G. Timoshenko and Yu. A. Kuznetsov, Colloids Surf., A 190, 135 (2001).

${ }^{35}$ V. A. Bloomfield, Biopolymers 31, 1471 (1991).

${ }^{36}$ S. Y. Park, D. Harries, and W. M. Gelbart, Biophys. J. 75, 714 (1998).

${ }^{37}$ K. Yoshikawa and Y. Matsuzawa, J. Am. Chem. Soc. 118, 929 (1996).

${ }^{38}$ Y. Matsuzawa, Y. Yonezawa, and K. Yoshikawa, Biochem. Biophys. Res. Commun. 225, 796 (1996).

${ }^{39}$ E. M. Lifshitz and L. P. Pitaevskii, Physical Kinetics (Pergamon, New York, 1981).

${ }^{40}$ K. Yoshikawa and Y. Matsuzawa, Physica D 84, 220 (1995). 